UTF 431

\title{
M-Theory and Stringy Corrections to Anti-de Sitter Black Holes and Conformal Field Theories
}

\author{
Marco M. Caldarelli* and Dietmar Klemm ${ }^{\dagger}$ \\ Università degli Studi di Trento, \\ Dipartimento di Fisica, \\ Via Sommarive 14 \\ 38050 Povo (TN) \\ Italia \\ and \\ Istituto Nazionale di Fisica Nucleare, \\ Gruppo Collegato di Trento, \\ Italia
}

\begin{abstract}
We consider black holes in anti-de Sitter space $\operatorname{AdS}_{p+2}(p=2,3,5)$, which have hyperbolic, flat or spherical event horizons. The $\mathcal{O}\left(\alpha^{\prime 3}\right)$ corrections (or the leading corrections in powers of the eleven-dimensional Planck length, in the case of M-theory compactifications) to the black hole metrics are computed for the various topologies and dimensions. We investigate the consequences of the stringy or M-theory corrections for the black hole thermodynamics. In particular, we show the emergence of a stable branch of small spherical black holes. Surprisingly, for any of the considered dimensions and topologies, the corrected thermodynamical quantities turn out to coincide with those calculated within a simplified approach, which uses only the unperturbed metric. We obtain the corrected Hawking-Page transition temperature for black holes with spherical horizons, and show that for $p=3$ this phase transition disappears at a value of $\alpha^{\prime}$ considerably smaller than that estimated previously by Gao and Li. Using the AdS/CFT correspondence, we determine the $S^{1} \times S^{3} \mathcal{N}=4$ SYM phase diagram for sufficiently large 't Hooft coupling, and show that the critical point at which the Hawking-Page
\end{abstract}

*email: caldarel@science.unitn.it

†email: klemm@science.unitn.it 
transition disappears (the correspondence point of Horowitz-Polchinski), occurs at $g_{Y M}^{2} N \approx 20.5$. The $d=4$ and $d=7$ black hole phase diagrams are also determined, and connection is made with the corresponding boundary CFTs. Finally, for flat and hyperbolic horizons, we show that the leading stringy or M-theory corrections do not give rise to any phase transition. However, if the horizon is compactified to a torus $T^{p}$ or to a quotient of hyperbolic space, $\mathbb{H}^{p} / \Gamma$, the appearance of light winding modes around non-contractible cycles signal new phase transitions, which in the toroidal case have previously been discussed by Barbón et al. . We comment on these phase transitions for SYM on $\mathbb{H}^{p} / \Gamma$ and on $T^{p}$, when the moduli of the torus are taken into account.

04.70.-s, 11.25.Hf, 04.60.-m, 04.65.+e 


\section{INTRODUCTION}

Black holes in anti-de Sitter space have turned out to be important tools to study large $N$ gauge theories. The connection between black hole physics and field theory is provided by the AdS/CFT correspondence [1 4], according to which supergravity on AdS spaces (times some compact manifold) is dual to certain superconformal field theories. An interesting application of this correspondence was the observation of Witten [3], who interpreted the Hawking-Page phase transition [5] from thermal five-dimensional AdS space at low temperature to a Schwarzschild-AdS black hole at high temperature, as a phase transition in the corresponding $d=4$ super Yang-Mills theory in the limit of large 't Hooft coupling. For the black hole, the free energy scales as $N^{2}$, whereas for thermal AdS space, it is of order one. On the Yang-Mills side, therefore, the low temperature phase is confining, whereas the high temperature phase is deconfining, the $N^{2}$ scaling representing the gluon degrees of freedom. This example feeds the hope that the AdS/CFT correspondence finally will help us to understand confinement in QCD [6] 8] (cf. also [9] for a review of the present status of the art).

Now, for AdS black holes, the presence of the negative cosmological constant makes it possible to circumvent Hawking's theorem forbidding non-spherical event horizon topology [10]. As a result, in AdS space one can have a large variety of black holes, whose event horizons are Einstein manifolds $\mathcal{M}$ with positive, negative, or zero scalar curvature [11] [17], the latter arising also as near-horizon limits of certain $p$-branes in supergravity theories [18]. If the event horizon is diffeomorphic to $\mathbb{R}^{p}$ ( $p$-brane solution), Witten [3] showed that there is no Hawking-Page phase transition, i. e. the system is always in the high temperature (black hole) phase. This feature was later shown by Birmingham [17] to be valid also for hyperbolic black holes, i. e. for those whose event horizons have negative scalar curvature. According to the AdS/CFT dictionary, this means that the corresponding field theory, which now lives on $S^{1} \times \mathcal{M}$, has no phase transition as a function of the temperature in the large $N$ limit, if $\mathcal{M}$ is hyperbolic or if $\mathcal{M} \approx \mathbb{R}^{p}$. In the $\mathbb{R}^{p}$ case, this is immediately clear from the fact that the circumference $\beta$ of the $S^{1}$ factor can be scaled out by conformal invariance, whereas for spherical black holes, leading to a CFT on $S^{1} \times S^{p}$, we can dispose of the circumference $\beta^{\prime}$ of $S^{p}$ to form the conformally invariant ratio $\beta / \beta^{\prime}$ [3]. For hyperbolic black holes, however, the absence of any phase transition at nonzero temperature is not so clear, as the curvature radius of $\mathcal{M}$ provides a characteristic scale.

If one wishes to go beyond the supergravity approximation, in order to study conformal field theories on the boundary of AdS, one has to calculate stringy corrections (or corrections in powers of the eleven-dimensional Planck length, in the case of M-theory) to the bulk spacetimes. This has been done in [19] for the D3-, M2-, and M5-branes. (Cf. also [20] for the case of D3-branes). The motivation for doing so was that the free energy of $d=4$ maximally supersymmetric $S U(N)$ gauge theory showed a seeming discrepancy of a factor $3 / 4$ between the calculations performed by using near-extremal D3-brane geometry, and the weak coupling limit. (Actually, this is not a contradiction, because the supergravity approximation is valid for strong ' $t$ Hooft coupling $g_{Y M}^{2} N$ ). The authors of [19] showed that the leading stringy corrections raise this factor 3/4. As far as the five-dimensional Schwarzschild-AdS black hole is concerned, stringy corrections to the thermodynamics have first been computed in [21], by using a simplified perturbation theory method. (Later, the results of [21] 
have been confirmed by a calculation of the $\alpha^{\prime}$-perturbed metric [22]). It was shown that these corrections lower the Hawking-page transition temperature. Furthermore, the authors of [21] argued that the Hawking-Page transition eventually disappears at sufficiently small coupling. This would then correspond to a critical point in the SYM phase diagram, where the first order Hawking-Page transition degenerates into a higher order phase transition. That point was conjectured to be identified with the correspondence point of Horowitz and Polchinski [23], which (in its simplest version) describes the transition from a highly excited string state to a black hole, when the characteristic curvature radius at the horizon becomes of order the string length. The entropy is in fact continuous at this correspondence point, like in a higher order phase transition. This continuity has been used in [23] to provide a statistical interpretation of the entropy of Schwarzschild black holes by string states.

Whereas the previous papers [19 22] treat the five-dimensional case, with event horizon either diffeomorphic to $\mathbb{R}^{3}$ or to $S^{3}$, we shall extend the computations of stringy- and Mtheory corrections to arbitrary event horizon topology, and also to the dimensions $d=4$ and $d=7$. In all cases, we determine the corrected metric, and compare the thermodynamical quantities obtained in this manner with those calculated within a perturbative approach, which uses the uncorrected metric. Quite interestingly, the corrected black hole entropy and free energy will turn out to coincide with the corresponding quantities computed with the perturbative technique. In five dimensions, this result was already obtained in [19] and [22] for the case of flat or spherical event horizons. It is surprising that it holds also for the hyperbolic case, which involves the subtraction of a nontrivial background in computing the action. This background, unlike the AdS vacuum used in the spherical or flat case, represents an extremal black hole which itself receives stringy corrections.

Furthermore, we shall see that some interesting new features arise, like the emergence of a stable branch of small spherical black holes. We will determine the behaviour of the Hawking-Page transition temperature in function of $\alpha^{\prime}$ for $p=3$ (or, alternatively, in function of $g_{Y M}^{2} N$ ). By using the simplified perturbative approach mentioned above, Gao and Li [21] were able to give a rough estimate for the critical value of $\alpha^{\prime}$ at which the Hawking-Page transition disappears. By extrapolating the $T_{c}=T_{c}\left(\alpha^{\prime}\right)$ curve to zero temperature, they obtained $\alpha_{c r i t}^{\prime} \approx 0.55 l^{2}$, where $l$ is the $\mathrm{AdS}_{5}$ radius. This value corresponds to $g_{Y M}^{2} N \approx 1.65$. We shall see below that a detailed analysis yields a disappearance of the Hawking-Page transition already at a critical value of $\alpha^{\prime}$ considerably smaller, namely $\alpha_{c r i t}^{\prime} \approx 0.16 l^{2}$, corresponding to $g_{Y M}^{2} N \approx 20.5$. In the phase diagram, this gives rise to a critical point (the Horowitz-Polchinski correspondence point), at which the first order transition degenerates to higher order. Besides, we show that there is a second critical value $g_{Y M}^{2} N \approx 33.4$ in the phase diagram, at which an additional phase of small black holes appears. Taking into account the different black hole or AdS phases, we will be able to derive the phase diagram of SYM on $S^{1} \times S^{3}$ for sufficiently large 't Hooft coupling. We also determine the phase structures of the $d=4$ and $d=7$ spherical black holes in M-theory, relevant for $\mathcal{N}=8$ SYM on $S^{1} \times S^{2}$ and an exotic $(0,2)$ CFT on $S^{1} \times S^{5}$ respectively.

On the other hand, in the hyperbolic case, it will turn out that the stringy corrections do not lead to the appearance of phase transitions, if the hyperbolic space is simply connected. If it is compactified, instead, strings can wrap around the non-contractible 1-cycles, and we shall briefly comment on the effect of light winding modes on the modification of the geometry and the possible appearance of phase transitions. In the flat case, $\mathcal{M} \approx \mathbb{R}^{p}$, as 
already remarked above, there can be no phase transition at any nonzero temperature. This changes however, if we compactify the $\mathbb{R}^{p}$ down to a torus. (For black holes and SYM on tori cf. 24,25]). The implications of these finite size effects on the emergence of phase transitions have been studied in [2]], and we will also give a short comment on the more general case when the torus is not symmetric, i. e. if one takes into account its moduli.

Finally, as further motivation to determine stringy corrections to black holes, we would like to mention that, apart from the possibility to study the thermodynamics of the corresponding conformal field theories on the boundary, corrections in $\alpha^{\prime}$ are also interesting on the black hole (bulk) side, from the point of view of a possible resolution of black hole singularities [27].

The remainder of this paper is organized as follows: To make it self-contained, we begin in section [1] by giving a short introduction into the geometry and thermodynamics of $d$-dimensional topological black holes. In [II, some AdS compactifications of string- and Mtheory are reviewed, with emphasis on non-trivial compactifications involving Hopf bundles over complex projective spaces. In IV, we give the leading corrections to the supergravity actions, and use a simple approach involving the unperturbed metric, to calculate the leading corrections to the black hole free energy and entropy. In section $\mathrm{\nabla}$, the corrected black hole metrics are determined in the various cases, and in $\nabla \mathbb{1}$ the resulting thermodynamical quantities are discussed and compared with those obtained by the method used in $\square$. Furthermore, the various phase diagrams are determined. In VII, we summarize our results, and discuss them with regard to conformal field theories.

\section{GEOMETRY AND THERMODYNAMICS OF TOPOLOGICAL BLACK HOLES}

One peculiarity of anti-de Sitter space consists in the fact that the negative cosmological constant allows for black holes with non-spherical event horizon topology. The first works in the four-dimensional case [11 [16] have been generalized by Birmingham [17] to arbitrary dimension. The general solution in $d$ dimensions is given by the metric

$$
d s^{2}=-f(r) d t^{2}+f^{-1}(r) d r^{2}+r^{2} h_{i j}(x) d x^{i} d x^{j},
$$

where the coordinates are labeled as $x^{\mu}=\left(t, r, x^{i}\right), i=1, \ldots, d-2$, and the metric $h_{i j}(x)$ describes the transverse manifold $\mathcal{M}^{(d-2)}$. For (I) to be a solution of Einstein's equations with cosmological constant

$$
\Lambda=-\frac{(d-1)(d-2)}{2 l^{2}},
$$

the manifold $\mathcal{M}^{(d-2)}$ must be Einstein,

$$
\mathcal{R}_{i j}=(d-3) k h_{i j},
$$

$\mathcal{R}_{i j}$ denoting the Ricci tensor of $h_{i j}$, and $k$ is a constant, which can be restricted to the values $k=0, \pm 1$ without loss of generality. $k=1,0,-1$ then corresponds to elliptic, flat, or hyperbolic horizons respectively. For the function $f$, one gets 


$$
f(r)=k-\frac{\eta}{r^{d-3}}+\frac{r^{2}}{l^{2}}
$$

where $\eta$ is an integration constant, which is related to the black hole mass. Note that the manifold $\mathcal{M}^{(d-2)}$ is not required to be of constant curvature, it merely has to be Einstein. In $d=6$, for instance, we could take $\mathcal{M}^{4} \approx S^{2} \times S^{2}$, which clearly can carry no metric of constant curvature, but it can carry an Einstein metric. If, on the other hand, $\mathcal{M}^{(d-2)}$ is of constant curvature (which we will assume in the following, as we want the solution with $\eta=0$ to be locally anti-de Sitter [17] Đ), it must be a quotient of $S^{d-2}, \mathbb{R}^{d-2}$, or $\mathbb{H}^{d-2}$ for $k=1, k=0$, or $k=-1$ respectively, where $\mathbb{H}^{d-2}$ denotes $(d-2)$-dimensional hyperbolic (Lobachevsky) space. This means that even in the case $k=1$ we are not restricted to the sphere, but we can also have lens spaces etc.

The spacetime with metric (1) has an event horizon at $r=r_{+}$, where $r_{+}$is the largest root of $f(r)=0$. It is interesting that for $k=-1$, one can also have black holes for negative values of the parameter $\eta$; the equation $f(r)=0$ has positive solutions for

$$
\eta \geq \eta_{0}=-\frac{2}{d-1}\left(\frac{d-3}{d-1}\right)^{\frac{d-3}{2}} l^{d-3} .
$$

For $\eta=\eta_{0}$, the black hole becomes extremal, with an event horizon at

$$
r_{+}=r_{0}=\left(\frac{d-3}{d-1}\right)^{\frac{1}{2}} l .
$$

For $k=-1$ and $\eta=0$, one has black holes which are locally AdS; they represent higherdimensional generalizations of the BTZ black hole 2. The thermodynamics of the black holes presented above, and their implications on the boundary conformal field theories have been examined in [17]. Requiring the absence of conical singularities in the euclidean metric yields the Hawking temperature

$$
T=\frac{(d-1) r_{+}^{2}+(d-3) k l^{2}}{4 \pi l^{2} r_{+}} .
$$

The mass is given by

$$
M=\frac{r_{+}^{(d-3)}}{\omega_{d}}\left(k+\frac{r_{+}^{2}}{l^{2}}\right)+\frac{\eta_{0}}{\omega_{d}} \delta_{k,-1},
$$

where

\footnotetext{
${ }^{1}$ Observe that if $\mathcal{M}^{(d-2)}$ is three-dimensional, the fact that it is Einstein implies that it is of constant curvature.

${ }^{2}$ Note that in the case $k=-1, \eta=0$, one has a black hole interpretation only if the space $\mathbb{H}^{d-2}$ has been compactified; the spacetime with topology $\mathbb{R}^{2} \times \mathbb{H}^{d-2}$ represents AdS seen by a uniformly accelerated observer, with $r=r_{+}=l$ denoting its acceleration horizon [16].
} 


$$
\omega_{d}=\frac{16 \pi G_{d}}{(d-2) V_{d-2}}
$$

Here $V_{d-2}$ denotes the volume of $\mathcal{M}^{(d-2)}$, which we assumed to be compact; otherwise one would have to define the mass per unit volume. The term proportional to the Kronecker delta in (8) corresponds to the correct choice of background, such that the Arnowitt-DeserMisner (ADM) mass is a positive, concave function of the black hole's temperature 16. This background is the one with $\eta=0$ (i. e. locally AdS) for $k=0,1$, but for $k=-1$, one has to choose the extremal black hole with $\eta=\eta_{0}$ as reference background [16]. The euclidean action reads

$$
I_{d}^{(0)}=\frac{V_{d-2} \beta}{16 \pi G_{d}}\left[-\frac{r_{+}^{d-1}}{l^{2}}+k r_{+}^{d-3}+2 \frac{d-2}{d-1}\left(\frac{d-3}{d-1}\right)^{\frac{d-3}{2}} l^{d-3} \delta_{k,-1}\right],
$$

yielding the entropy

$$
S=\frac{V_{d-2}}{4 G_{d}} r_{+}^{d-2}
$$

In (10) and (11), the horizon radial coordinate $r_{+}$can be expressed as a function of the temperature via

$$
r_{+}(T)=\frac{2 \pi l^{2} T}{d-1}\left[1 \pm\left(1-\frac{(d-1)(d-3) k}{(2 \pi T l)^{2}}\right)^{\frac{1}{2}}\right]
$$

Here the lower sign makes sense only in the spherical $(k=1)$ case, where two different values of $r_{+}$are possible for a given temperature, the branch of small black holes being unstable [5]. The free energy of the black hole as well as that of the boundary conformal field theory is then given by $F=I_{d}^{(0)} / \beta$, and is easily seen to scale as $V_{d-2} T^{d-1}$ in the limit of high temperatures, independently of the curvature $k$. (In the planar case, $k=0$, this scaling law is valid for all temperatures).

\section{TOPOLOGICAL BLACK HOLES IN STRING- AND M-THEORY}

In this section we shall review how the black holes described above arise as solutions of the low energy effective actions of M-theory or string theory for special values of $d$. We shall first see how the topological black holes, for $d=4$, emerge in low-energy limit of M-theory and type IIA string theory as exact solutions of $D=11, N=1$ supergravity and type IIA supergravity respectively. Emparan [18] considered $d=4$ topological black holes in their M-theory context. The solution of the corresponding low-energy effective action, i. e. of $D=11$ supergravity, reads

$$
d s^{2}=-f(r) d t^{2}+f^{-1}(r) d r^{2}+r^{2} d \sigma_{k}^{2}+4 l^{2} d \Omega_{7}^{2}
$$

where $f(r)=k-\eta / r+r^{2} / l^{2}, d \sigma_{k}^{2}$ is the metric on $S^{2}$, the two-torus or $\mathbb{H}^{2}$ for $k=1,0,-1$ respectively, and $d \Omega_{7}^{2}$ denotes the metric on $S^{7}$. The four-form field strength is $F_{\mu \nu \rho \sigma}=$ 
(3/l) $\epsilon_{\mu \nu \rho \sigma}$, and corresponds to the Freund-Rubin ansatz for the eleven dimensional manifold. The associated 3 -form potential $A_{[3]}$ can be chosen to be $A_{t \theta \phi}=-3 r / l$, the other components being zero. These objects also describe the near-horizon limit of non-extremal M2-branes wrapped around compact surfaces of arbitrary genus.

Proceeding with a Kaluza-Klein compactification on a circle of M-theory, one obtains type IIA superstring theory [28]. In particular, compactification of (13) leads to a topological black hole in type IIA supergravity. One observes first that the odd-dimensional sphere $S^{2 n-1}$ can be written as a $U(1)$ bundle over $\mathbb{C P}^{n-1}$; it is then possible to perform the Kaluza-Klein reduction on the $U(1)$ direction of the Hopf fibres [29]. Hence, we write the $S^{7}$ factor of the metric (13) as

$$
d \Omega_{7}^{2}=d \Sigma_{6}^{2}+\left(d z+\overline{\mathcal{A}}_{M} d z^{M}\right)^{2},
$$

where $d \Sigma_{6}^{2}$ is the standard Fubini-Study metric on $\mathbb{C P}^{3}$, and the Kaluza-Klein vector potential $\overline{\mathcal{A}}$ has field strength $\overline{\mathcal{F}}$ given by $\overline{\mathcal{F}}=2 J$, where $J$ is the Kähler form on $\mathbb{C P}^{3}$. The coordinate on the fiber is $z$ and has period $4 \pi$, while the coordinates $z^{M}, M=1 \ldots 6$, parametrize $\mathbb{C P}^{3}$. We can now compactify M-theory along the $S^{1}$ direction parametrized by the coordinate $z$. The resulting field configuration will solve the equations of motion of type IIA supergravity; the corresponding metric is described by the line element

$$
d s_{I I A}^{2}=2^{1 / 4}\left(-f(r) d t^{2}+\frac{d r^{2}}{f(r)}+r^{2} d \sigma_{k}^{2}+4 l^{2} d \Sigma_{6}^{2}\right),
$$

and represents a direct product of a four-dimensional black hole and $\mathbb{C P}^{3}$. The dilaton is constant, and assumes the value $\phi=\frac{3}{2} \ln 2$. The Ramond/Ramond (RR) one-form $A_{[1]}$ can be read directly from the mixed components $g_{\mu z}$; it is non-vanishing only on $\mathbb{C P}^{3}$, $A_{[1] M}=l \overline{\mathcal{A}}_{M}$. The remaining fields of IIA supergravity, the Neveu-Schwarz/Neveu-Schwarz (NSNS) two-form $B_{[2]}$ and the RR three-form $C_{[3]}$, are determined by dimensional reduction of the three-form $A_{[3]}$ of $D=11$ supergravity,

$$
A_{[3]}=C_{[3]}+B_{[2]} \wedge l d z,
$$

from which we deduce that the antisymmetric tensor field $B_{[2]}$ vanishes while the RR threeform coincides with the three-potential of $D=11$ supergravity, $C_{[3] t \theta \phi}=-\frac{3 r}{l}$. Note that the compactification of IIA supergravity on $\mathbb{C P}^{3}$ breaks some supersymmetry; the reason is that some Killing spinors are described by D0-branes, and the full supersymmetry is restored only at type IIA superstring theory level [30].

Modifying slightly the Freund-Rubin ansatz we obtain seven-dimensional topological black holes in $\mathrm{D}=11$ supergravity. We ask again the eleven dimensional manifold to be a direct product $\mathcal{M}_{7} \times \mathcal{M}_{4}$, but now we take $\mathcal{M}_{7}$ lorentzian and $\mathcal{M}_{4}$ euclidean. We require the field $F$ to be non-vanishing only on $\mathcal{M}_{4}$, where it takes the value $F_{m n p q}=(6 / l) \epsilon_{m n p q}$. This yields an Einstein space $\mathcal{M}_{4}$ of positive curvature, and we take it to be the four-sphere, which is maximally symmetric. Other choices are possible, at the cost of breaking some of the supersymmetries. The $\mathcal{M}_{7}$ factor is an Einstein space of negative curvature, for example any of the seven-dimensional topological black holes (11). As a result we have

$$
d s^{2}=-f(r) d t^{2}+f^{-1}(r) d r^{2}+r^{2} h_{i j}^{(5)}(x) d x^{i} d x^{j}+\frac{1}{4} l^{2} d \Omega_{4}^{2},
$$


where $f(r)=k-\eta / r^{4}+r^{2} / l^{2}$, and $h_{i j}^{(5)}$ is the metric of a five-dimensional compact Einstein space of positive, zero or negative curvature according to the sign of $k$. Observe that we cannot obtain a seven dimensional black hole in IIA supergravity from this solution, because $S^{4}$ has even dimension and hence cannot be written as a $U(1)$ bundle.

Let us turn now to five dimensional topological black holes. In type IIB supergravity there is a self-dual five-form $F$ that can be used for a Freund-Rubin like ansatz [31]. Taking

$$
F_{\mu_{1} \ldots \mu_{5}}=\frac{4}{l} \epsilon_{\mu_{1} \ldots \mu_{5}}, \quad F_{m_{1} \ldots m_{5}}=-\frac{4}{l} \epsilon_{m_{1} \ldots m_{5}}
$$

and all the other fields zero, the ten-dimensional spacetime can become the direct product of a five-dimensional topological black hole and a five-dimensional positive curvature Einstein space, which represents a solution of the IIB supergravity equations of motion. The metric reads

$$
d s^{2}=-f(r) d t^{2}+f^{-1}(r) d r^{2}+r^{2} h_{i j}^{(3)}(x) d x^{i} d x^{j}+l^{2} d \Omega_{5}^{2},
$$

where $f(r)=k-\eta / r^{2}+r^{2} / l^{2}$, and $h_{i j}^{(3)}$ denotes the metric of a three-dimensional compact Einstein space of positive, zero or negative curvature, according to the sign of $k$.

Furthermore, if the compact space is $S^{5}$, we can untwist it [29], and obtain a five-dimensional black hole times a compact space $\mathbb{C P}^{2} \times U(1)$ as solution of type IIA supergravity. Let us see in detail how this works. The sphere $S^{5}$ is a $U(1)$ bundle over $\mathbb{C P}^{2}$ and its metric is

$$
d s^{2}\left(S^{5}\right)=l^{2} d \Sigma_{4}^{2}+l^{2}(d z+\overline{\mathcal{A}})^{2},
$$

where $d \Sigma_{4}^{2}$ is the metric on the "unit" $\mathbb{C P}^{2}$, and $d \overline{\mathcal{A}}=2 J, J$ being the Kähler form on $\mathbb{C P}^{2}$. Now, performing a T-duality along $z$, we obtain a solution of type IIA supergravity [32]. The dual metric reads

$$
d s_{I I A}^{2}=-f(r) d t^{2}+f^{-1}(r) d r^{2}+r^{2} h_{i j}^{(3)}(x) d x^{i} d x^{j}+l^{2} d \Sigma_{4}^{2}+l^{2} d z^{2},
$$

and is of the form $\mathrm{TBH}_{5} \times \mathbb{C P}^{2} \times S^{1}$. The RR 4-form of IIB theory is mapped by T-duality on the RR 3-form of IIA theory,

$$
d C_{[3]}=4 l^{3} \Sigma_{4}
$$

where $\Sigma_{4}$ denotes the volume-form on $\mathbb{C P}^{2}$, and the Kaluza-Klein vector component of the ten-dimensional IIB metric is mapped on the 2 -form $B_{[2]}$ of the NSNS sector of IIA supergravity,

$$
d B_{[2]}=2 l J \wedge d z
$$

while the RR 1-form and the dilaton $\phi$ vanish. Observe that $\mathbb{C P}^{2}$ does not admit any spin structure. As a consequence the spectrum of Kaluza-Klein excitations in the $\mathbb{C P}^{2} \times S^{1}$ compactification of type IIA supergravity contains no fermions.

Finally, the oxidation of the $\mathrm{TBH}_{5} \times \mathbb{C P}^{2} \times S^{1}$ solution of IIA supergravity yields a fivedimensional topological black hole solution of $\mathrm{D}=11$ supergravity 


$$
\begin{aligned}
d s_{11}^{2} & =d s_{T B H_{5}}^{2}+l^{2} d \Sigma_{4}^{2}+d z_{1}^{2}+d z_{2}^{2}, \\
F & =4 l^{2} \Sigma_{4}-2 l J \wedge d z_{1} \wedge d z_{2},
\end{aligned}
$$

which is of the form $\mathrm{TBH}_{5} \times \mathbb{C P}^{2} \times \mathrm{T}^{2}$.

In fact, more general five-dimensional AdS black hole solutions can be found, of the form $T B H_{5} \times \mathcal{M}^{6}$, where $\mathcal{M}^{6}$ is Einstein-Kähler and $\mathrm{F}$ is the product of the Kähler form with itself [33. In the case of M-theory compactifications to $d=4,7$, one can choose less symmetric compactification spaces, substituting for example the spheres with lens spaces, or taking other Einstein manifolds. The resulting solutions have less supersymmetries, and could show relevant to study CFT with less than maximal supersymmetry 34].

In conclusion, we have obtained several topological black hole solutions. In $\mathrm{D}=11$ supergravity we have $\mathrm{TBH}_{4} \times S^{7}, \mathrm{TBH}_{7} \times S^{4}$ and $T B H_{5} \times \mathbb{C P}^{2} \times T^{2}$ black holes, in type IIA supergravity we obtained $\mathrm{TBH}_{4} \times \mathbb{C P}^{3}$ and $T B H_{5} \times \mathbb{C P}^{2} \times S^{1}$ black holes, while in IIB supergravity we have the $T B H_{5} \times S^{5}$ black holes.

\section{STRINGY AND M-THEORY CORRECTIONS TO FREE ENERGY AND ENTROPY FROM THE UNPERTURBED METRIC}

\section{A. The Modified Supergravity Actions}

Our aim is to determine effects of massive string states on the black hole geometry. In the low energy effective action, these massive string states manifest themselves as higher derivative curvature terms. In type IIB supergravity, the lowest correction is of order $\alpha^{\prime 3} R^{4}$, where $R$ denotes the Riemann tensor. Taking this into account, the string-corrected IIB supergravity action reads in Einstein frame [35, 36]

$$
I_{10}=-\frac{1}{16 \pi G_{10}} \int d^{10} x \sqrt{g}\left[R-\frac{1}{2}(\partial \phi)^{2}-\frac{1}{4 \cdot 5 !}\left(F_{5}\right)^{2}+\ldots+\gamma e^{-\frac{3}{2} \phi} W+\ldots\right]
$$

where

$$
\gamma=\frac{1}{8} \zeta(3)\left(\alpha^{\prime}\right)^{3}
$$

and the dots stand for terms depending on further antisymmetric tensor field strengths and dilaton derivatives. Due to a field redefinition ambiguity [36, 37], there exists a scheme where $W$ depends only on the Weyl tensor,

$$
W=C^{h m n k} C_{p m n q} C_{h}^{r s p} C_{r s k}^{q}+\frac{1}{2} C^{h k m n} C_{p q m n} C_{h}^{r s p} C_{r s k}^{q} .
$$

For eleven-dimensional supergravity, the situation is somewhat different, as there is no parameter $\alpha^{\prime}$ in M-theory. However, there are corrections in powers of the Planck length $\ell_{11}$, leading to the effective action (cf. [19] and references therein)

\footnotetext{
${ }^{3}$ We impose self-duality of the five-form field strength $F_{5}$ after the equations of motion are derived.
} 


$$
I_{11}=-\frac{1}{2 \kappa_{11}^{2}} \int d^{11} x \sqrt{-g}\left[R+2 \kappa_{11}^{4 / 3} \xi W+\ldots\right]
$$

where $\xi=2 \pi^{2} / 3$, and the eleven-dimensional Newton constant reads in terms of the Planck length $\ell_{11}$

$$
8 \pi G_{11}=\kappa_{11}^{2}=2^{4} \pi^{5} \ell_{11}^{9}
$$

As will be argued shortly, for the problem at hand one can use the four- or seven-dimensional analogue of (28) for the term $W$ occuring in (29). For further purpose, it will be useful to define also in eleven dimensions an expansion parameter $\gamma$, which is now given by $\gamma=2 \kappa_{11}^{4 / 3} \xi$, so that the $W$-dependent term in the corrected actions has the prefactor $\gamma$ in both ten and eleven dimensions.

\section{B. Free Energy and Entropy}

In order to get a first clue of the string- or M-theory corrections to thermodynamical quantities like free energy or black hole entropy, one can plug the unperturbed metric into the expression (28), and then calculate the corrected action. This approach corresponds essentially to first order perturbation theory. It was used in 19 to calculate $\alpha^{\prime}$ corrections to the throat approximation of D3-branes, and Planck length corrections to that of M2and M5-branes, in order to determine the free energy behaviour of conformal field theories beyond the supergravity approximation in the AdS/CFT correspondence. The authors of 119 showed that in the D3-brane case, this simple procedure yields exactly the same result as a calculation involving the perturbed metric. For the five-dimensional $k=1$ SchwarzschildAdS black hole, Gao and Li [21] used the same approach to show that the Hawking-Page phase transition temperature is lowered by the first $\alpha^{\prime}$ correction. Later, Landsteiner 22] computed explicitely the perturbed black hole metric, and obtained the same black hole entropy as that supplied by the perturbative approach of 21. In section VI we will see that this coincidence extends also to the hyperbolic case $(k=-1)$, and to the dimensions $d=4,7$. This result is rather puzzling, and it would be interesting to get a better physical understanding of it.

In the following, we will calculate the corrected action for the $d$-dimensional topological black hole spacetimes (11), where we shall concentrate on the cases $d=p+2=4,5,7$, corresponding to the well-known AdS compactifications of M-theory or of type IIB string theory. In calculating the term (28) quartic in the Weyl tensor, one actually should take the full ten- or eleven-dimensional metric, i. e. the product of (1) and the metric on a suitably chosen sphere. In the case of D3-branes in the throat approximation, the full ten-dimensional expression for $W$ coincides with the one calculated using only the five-dimensional part without five-sphere [19]. This fact is related to a vanishing scalar curvature of the full tendimensional metric [19]. An argument which justifies the use of the $(p+2)$-dimensional analogue of (28) also in the case of M-branes down the throat, was given in 19. The same argument applies also if the branes are wrapped on Einstein spaces $\mathcal{M}^{p}$, i. e. in our case. Therefore, in the following, we will use the $d=p+2$ dimensional analogue of (28), and compute it for the metric (11). $W$ is then given by 


$$
W=d_{p+2}\left[\frac{\eta(d-2)(d-1)}{2 r^{d-1}}\right]^{4}
$$

where we defined the coefficients

$$
d_{4}=\frac{1}{18}, \quad d_{5}=\frac{5}{36}, \quad d_{7}=\frac{292}{1125} .
$$

Upon dimensional reduction on a suitable sphere, the correction to the actions (26) resp. (29) becomes

$$
\delta I_{d}=-\frac{1}{16 \pi G_{d}} \int d^{d} x \sqrt{g_{d}} \gamma W, \quad d=4,5,7
$$

where

$$
\begin{aligned}
& \frac{1}{16 \pi G_{5}}=\frac{\operatorname{Vol}\left(S^{5}\right)}{16 \pi G_{10}}=\frac{\pi^{3} l^{5}}{16 \pi G_{10}}, \\
& \frac{1}{16 \pi G_{4}}=\frac{\operatorname{Vol}\left(S^{7}\right)}{16 \pi G_{11}}=\frac{\pi^{4}(2 l)^{7} / 3}{16 \pi G_{11}}, \\
& \frac{1}{16 \pi G_{7}}=\frac{\operatorname{Vol}\left(S^{4}\right)}{16 \pi G_{11}}=\frac{8 \pi^{2}(l / 2)^{4} / 3}{16 \pi G_{11}} .
\end{aligned}
$$

(Note that in our conventions the radii of the four- and seven spheres are given by $l / 2$ and $2 l$ respectively). In (33), $\gamma$ is defined by $\gamma=\zeta(3)\left(\alpha^{\prime}\right)^{3} / 8$ for $d=5$, and by $\gamma=$ $4 \pi^{2} \kappa_{11}^{4 / 3} / 3$ for $d=4,7$. Plugging (31) into (33), one can compute the stringy or M-theory corrections. In doing so, one has to take care of an important subtlety. In the calculation of the unperturbed action, one performs a subtraction of a suitably chosen background in order to cancel divergences. Now, as already observed in [1], for the cases $k=0$ or $k=1$ this background is (at least locally) anti-de Sitter space, which receives no corrections, as it has $\eta=0$. For the hyperbolic black hole $(k=-1)$ however, the correct background is the extremal black hole with $\eta=\eta_{0}$ (cf. (5)). In calculating the corrected action, we must therefore subtract the background value, which gives a nonvanishing contribution in the hyperbolic case, as can be seen from (31). In this way, we get

$$
\delta I_{d}=-\frac{\gamma V_{d-2} \beta}{16 \pi G_{d}} d_{p+2} \frac{(d-2)^{4}(d-1)^{3}}{3}\left[\frac{r_{+}^{d-9}}{16 l^{8}}\left(k l^{2}+r_{+}^{2}\right)^{4}-\delta_{k,-1} \frac{l^{d-9}}{(d-1)^{4}}\left(\frac{d-3}{d-1}\right)^{\frac{d-9}{2}}\right],
$$

where $r_{+}$is a function of the temperature according to (12). The correction to the free energy is then $\delta F_{d}=\delta I_{d} / \beta$, which again scales as $V_{d-2} T^{d-1}$ for high temperatures, independently of the curvature $k$. One immediately checks (after adapting the conventions), that for $k=0$, (35) reproduces the results of [19], and for $d=5, k=1$ it reduces to the corrected action obtained in [21]. We further observe that for $k=0,1$ the correction to the action is always negative. The entropy correction can be computed by $S=-\frac{\partial F}{\partial T}$, which yields

$$
\begin{aligned}
\delta S_{d}= & \frac{\gamma}{G_{d}} d_{p+2} \frac{(d-2)^{4} \pi^{3}}{3(d-3)}\left(\frac{2 \pi l^{2}}{d-1}\right)^{d-5} V_{p} T^{p}\left[1+(1-x)^{\frac{1}{2}}\right]^{d-5} \\
& \cdot\left[\frac{d-2}{d-3}-\frac{1}{d-3}(1-x)^{\frac{1}{2}}\right]^{3}\left[3+(d-6)(1-x)^{-\frac{1}{2}}\right]
\end{aligned}
$$


where we defined

$$
x=\frac{(d-1)(d-3) k}{(2 \pi T l)^{2}} .
$$

Note that the $k=-1, \eta=0$ locally AdS black holes, which have Hawking temperature $T=1 /(2 \pi l)$, do not receive any stringy or M-theory corrections to their entropy up to order $\alpha^{\prime 3}$. (At least not in this simplified calculation, but we shall see later that a computation involving the perturbed metric gives the same result; besides, for $\eta=0$ the metric does not receive any corrections). This suggests that maybe they represent exact solutions of stringor M-theory, similarly to the BTZ black hole, which has been shown to be an exact solution of string theory by Horowitz and Welch 38.

\section{CORRECTED BLACK HOLE METRICS}

In this section we shall compute the corrections to the metric of the $T B H_{4} \times S^{7}$ and $T \mathrm{BH}_{7} \times S^{4}$ black holes in M-theory and of the $T B H_{5} \times S^{5}$ solution in type IIB superstring theory. We shall proceed in a unified way, leaving the dimension $d$ of the black hole arbitrary, and carrying out the perturbative expansion in $\gamma$, defined in section IVA.

To compute the perturbed solution, following [19], we write the reduced action for a metric of the form

$$
d s^{2}=H(r)^{2}\left(K^{2}(r) d t^{2}+P^{2}(r) d r^{2}+l^{2} d \sigma_{k}^{2}\right)
$$

where $d \sigma_{k}^{2}$ is the unit metric of the transverse elliptic, flat or hyperbolic manifold for $k=1,0$ and -1 respectively. Using the symmetry of the ansatz, we can reduce the corrected actions (26) and (29) to one dimension in a unified way, and we obtain the effective action t

$$
I=-\frac{\beta V_{d-2}}{16 \pi G_{d}} \int_{r_{+}}^{\infty} \sqrt{g}(R-2 \Lambda+\gamma W) d r,
$$

where the expansion parameter $\gamma$ is given in section $\mathbb{D A}$ for IIB superstring theory and M-theory, $\beta$ is the period in euclidean time, $V_{d-2}$ is the volume of the (unit) transverse manifold and $\Lambda$ is the cosmological constant defined in (2). In the IIB supergravity case, the dilaton $\phi$ appears in the full action (26). However on the unperturbed solution it vanishes, and the leading order perturbations of the dilaton do not mix with the metric perturbations. Hence, in computing the corrections to the metric, we shall simply forget about the dilaton;

\footnotetext{
${ }^{4}$ For the subtleties concerning the self-duality of $F_{5}$ in the IIB supergravity case, we refer to [19]. Furthermore, a more general ansatz should actually be used, allowing also the radius of the compactification space to vary [20]. However, in [19] an argument is given that shows that such a generalization does not yield additional corrections to the free energy; this has been explicitly verified in some cases in [20,22]. Hence we shall use the simplified ansatz with fixed compactification space radius in the following.
} 
we will add some comments on its leading order perturbation at the end of this section. The invariant $W$ can be computed from the Weyl tensor and reads

$$
W=\frac{d_{p+2}}{H^{8}}\left[\frac{1}{K P}\left(\frac{K^{\prime}}{P}\right)^{\prime}-\frac{k}{l^{2}}\right]
$$

where the primes denote derivatives with respect to $r$. To simplify the action, it is convenient to set 19]

$$
H(r)=\frac{r}{l}, \quad K(r)=e^{a(r)+4 b(r)}, \quad P(r)=e^{b(r)} .
$$

Substituting the ansatz in the action we obtain

$$
\begin{aligned}
\sqrt{g}(R-2 \Lambda) & =-2 r^{d-3} e^{a+3 b}\left[r\left(a^{\prime \prime}+4 b^{\prime \prime}\right)+(d-1)\left(a^{\prime}+3 b^{\prime}\right)+r a^{\prime 2}+12 r b^{\prime 2}\right. \\
& \left.+7 r a^{\prime} b^{\prime}+\frac{(d-4)(d-1)}{2 r}\right]+(d-2) \frac{r^{d-2}}{l^{2}}\left[(d-3) k+(d-1) \frac{r^{2}}{l^{2}}\right] e^{a+5 b}
\end{aligned}
$$

for the zero order term, and

$$
\sqrt{g} W=d_{p+2} \frac{l^{6}}{r^{8-d}} e^{a-3 b}\left(a^{\prime \prime}+4 b^{\prime \prime}+a^{2}+7 a^{\prime} b^{\prime}+12 b^{\prime 2}-\frac{k}{l^{2}} e^{2 b}\right)^{4}
$$

for the first corrections to the lagrangian. The Euler-Lagrange equations corresponding to the reduced action yield the equations of motion for $a(r)$ and $b(r)$,

$$
\begin{array}{r}
21-5 d-2 r a^{\prime}+\left[\frac{5 r^{2}}{l^{2}}\left(k(d-3)+(d-1) \frac{r^{2}}{l^{2}}\right)+\frac{\gamma}{l^{6}} w_{b}(r)\right] e^{2 b}=0 \\
5-d+2 r b^{\prime}+\left[\frac{r^{2}}{l^{2}}\left(k(d-3)+(d-1) \frac{r^{2}}{l^{2}}\right)+\frac{\gamma}{l^{6}} w_{a}(r)\right] e^{2 b}=0
\end{array}
$$

where we have defined

$$
\begin{aligned}
& w_{a}(r)=-\frac{9 \eta^{3}}{4 l^{4} r^{8}}\left(56 r^{3}+64 k l^{2} r-67 l^{2} \eta\right), \\
& w_{b}(r)=-\frac{9 \eta^{3}}{4 l^{4} r^{8}}\left(224 r^{3}+264 k l^{2} r-279 l^{2} \eta\right) .
\end{aligned}
$$

We look now for first-order corrections to the metric. To this end, we first expand the functions $a(r)$ and $b(r)$ in $\gamma$,

$$
a(r)=a_{0}(r)+\gamma a_{1}(r)+\ldots, \quad b(r)=b_{0}(r)+\gamma b_{1}(r)+\ldots,
$$

where $a_{0}$ and $b_{0}$ are determined by the unperturbed solution,

$$
a_{0}(r)=\frac{1}{2} \ln \left[\frac{r^{6}}{l^{6}} f^{5}(r)\right], \quad b_{0}(r)=-\frac{1}{2} \ln \left[\frac{r^{2}}{l^{2}} f(r)\right],
$$


$f(r)$ being the lapse function defined in (身). Obviously, $a_{0}$ and $b_{0}$, which describe the unperturbed AdS black holes, solve the equations of motion (44) at zero order in $\gamma$. Expanding the equations to first order in $\gamma$ and solving them, one finds the $\mathcal{O}(\gamma)$ corrections to the metric,

$$
\begin{aligned}
a_{1}(r)= & -\frac{(d-1)^{3}(d-2)^{2}}{96 l^{2} r^{3 d-5} f(r)} d_{p+2} \eta^{3}\left[-104(2 d-1)-16(13 d-14) \frac{k l^{2}}{r^{2}}+9(17 d-6) \frac{l^{2} \eta}{r^{d-1}}\right. \\
& \left.+5\left(24(2 d-1)+48(d-1) \frac{k l^{2}}{r_{+}^{2}}-(37 d-14) \frac{l^{2} \eta}{r_{+}^{d-1}}\right)\left(\frac{r}{r_{+}}\right)^{2 d-2}\right]
\end{aligned}
$$

and

$$
\begin{aligned}
b_{1}(r)= & \frac{(d-1)^{3}(d-2)^{2}}{96 l^{2} r^{3 d-5} f(r)} d_{p+2} \eta^{3}\left[-24(2 d-1)-48(d-1) \frac{k l^{2}}{r^{2}}+(37 d-14) \frac{l^{2} \eta}{r^{d-1}}\right. \\
& \left.+\left(24(2 d-1)+48(d-1) \frac{k l^{2}}{r_{+}^{2}}-(37 d-14) \frac{l^{2} \eta}{r_{+}^{d-1}}\right)\left(\frac{r}{r_{+}}\right)^{2 d-2}\right] .
\end{aligned}
$$

The integration constants in $a_{1}(r)$ and $b_{1}(r)$ have been chosen such that the corrections vanish asymptotically, where the corrected metric must match the locally AdS metric. Furthermore, the position of the horizon is not modified by the corrections, which are finite for $r \rightarrow r_{+}$.

It is interesting to note that the $\gamma$-corrections vanish for $\eta=0$. This becomes plausible recalling that in this case the spacetimes are (at least) locally AdS. Using supersymmetry arguments, it was argued in [39] that $\mathrm{AdS}_{4} \times S^{7}, \mathrm{AdS}_{7} \times S^{4}$ or $\mathrm{AdS}_{5} \times S^{5}$ are exact vacua of M-theory or IIB string theory respectively. In the case $\eta=0, k=-1$ these arguments do not apply anymore, as spacetime represents now a black hole with Hawking temperature $T=1 /(2 \pi l)$, if the transverse space $\mathbb{H}^{p}$ has been compactified. Clearly a black hole with non-vanishing temperature cannot be supersymmetric [10]. Presumably, the $\eta=0, k=-1$ black holes are nevertheless exact solutions of string theory in much the same way as the BTZ black hole [38].

Finally, in the IIB supergravity case, one should also consider the corrections to the dilaton field. Reducing the complete action (26) to $d=5$ dimensions, one obtains the equations of motion for the leading order corrections $\phi_{1}(r)$ to the dilaton, $\left(\sqrt{g} \phi_{1}^{\prime}\right)^{\prime}=3 \gamma \sqrt{g} W$, and the corrections to the dilaton can be chosen to be

$$
\phi_{1}(r)=\gamma \int_{+\infty}^{r} \frac{45 \eta^{4}}{2 r_{+}^{12}}\left[\frac{r^{12}-r_{+}^{12}}{r^{15} f(r)}\right] d r .
$$

This integral can be performed analytically, but the result is quite complicated and we shall not need it in the following. In fact, the result can be found in [19] in the $k=0$ case and in [22] for $k=1$; in the hyperbolic case the result is quite similar. The relevant point is that the solution is regular everywhere, and the dilaton, as well as the string coupling $g_{s}$, decreases towards the horizon. 


\section{THERMODYNAMICS OF THE PERTURBED SOLUTION}

Having now the corrections to the metric, we shall analyse their effects on the thermodynamics, and compare the corrected free energy with the one obtained from the unperturbed solution in section [IV. The temperature of the perturbed black hole is obtained from the absence of conical singularities in the euclidean section,

$$
\begin{aligned}
T\left(r_{+}\right)= & \frac{(d-1) r_{+}^{2}+(d-3) k l^{2}}{4 \pi l^{2} r_{+}}-\gamma d_{p+2} \frac{(d-2)^{2}(d-1)^{3}}{192 \pi r_{+}^{7}}\left(k+\frac{r_{+}^{2}}{l^{2}}\right)^{3} \\
& \times\left[\left(d^{2}+d-18\right) k-(d-1)(14-d) \frac{r_{+}^{2}}{l^{2}}\right]+\mathcal{O}\left(\gamma^{2}\right) .
\end{aligned}
$$

The first term of (52) is the temperature of the unperturbed black hole (7), and the second term comes from the order $\gamma$ correction to the metric. For the toroidal black hole we have $k=0$ and the temperature reads

$$
T_{k=0}\left(r_{+}\right)=\frac{(d-1) r_{+}}{4 \pi l^{2}}\left(1+\frac{\gamma d_{p+2}}{48 l^{6}}(d-1)^{3}(d-2)^{2}(14-d)\right)+\mathcal{O}\left(\gamma^{2}\right) .
$$

The behaviour of the temperature as a function of the position of the horizon remains linear, but the coefficient is increased by the $\gamma$-corrections. In the spherical and hyperbolic cases, the temperature approaches asymptotically (53).

One can invert (52) and obtain the black hole radius $r_{+}$as a function of the temperature $T$. We use the unperturbed radius $r_{+(0)}$ defined in $(12)$ as

$$
r_{+(0)}(T)=\frac{2 \pi l^{2} T}{d-1}[1+\sqrt{1-x}]
$$

where $x$ is given by (37). The radius of the horizon is then

$$
\begin{aligned}
r_{+}(T)=r_{+(0)}(T) & {\left[1+\gamma \frac{d_{p+2}}{48 l^{6}}(d-2)^{2}(d-1)^{3} \frac{\left(r_{+(0)}^{2}(T)+k l^{2}\right)^{3}}{r_{+(0)}^{6}(T)}\right.} \\
& \left.\times \frac{(d-1)(d-14) r_{+(0)}^{2}(T)+\left(d^{2}+d-18\right) k l^{2}}{(d-1) r_{+(0)}^{2}(T)-(d-3) k l^{2}}\right] .
\end{aligned}
$$

We now wish to compute the corrected euclidean action of the black hole, as well as the corrected free energy. As usual in AdS space, the surface terms of the action do not contribute, but there is an infrared divergence coming from the bulk contribution. This is regularized setting a cut-off in the radial integration and subtracting the action of an appropriate background. As mentioned in section III, in the spherical and toroidal cases the appropriate background is the metric with $\eta=0$. We have shown that this background receives no correction. The hyperbolic case, however, is more subtle, as its background (5)

\footnotetext{
${ }^{5}$ In the $k=1$ case, $r_{+}(T)$ describes only the branch of larger black holes.
} 
gets corrections in $\gamma$. In fact, as can be readily verified from Eq. (52), its formal temperature becomes negative. The right background to subtract is the one whose corrected temperature (52) vanishes. The position of the horizon of this corrected black hole is obtained setting $T=0$ in Eq. (55),

$$
r_{+}(T=0)=l \sqrt{\frac{d-3}{d-1}}\left[1+\gamma \frac{d_{p+2}}{2 l^{6}} \frac{(d-2)^{2}(d-1)^{3}}{(d-3)^{4}}(3 d-10)\right] .
$$

The regularized action then reads

$$
I_{\text {reg }}=\beta I(R)-\beta^{\prime} I_{0}(R),
$$

where $I_{0}(R)$ is the action evaluated on the appropriate background with the radial cut-off at $R$. The periodicity in the euclidean time of the background $\beta^{\prime}$ must match that of the black hole, in such a way that the proper length of the circle which it parametrizes at radius $R$ is the same as for the black hole [3]. This yields

$$
\beta^{\prime}=\beta\left[1+\frac{\left(\eta_{0}-\eta\right) l^{2}}{2 R^{(d-1)}}+\mathcal{O}\left(\frac{1}{R^{(d+1)}}\right)\right] .
$$

At this point, we can safely eliminate the cut-off from (57) by sending $R$ to infinity, obtaining the black hole action

$$
\begin{aligned}
I=-\frac{V_{d-2} \beta}{16 \pi G_{d}} & {\left[\frac{r_{+}^{d-1}}{l^{2}}-k r_{+}^{d-3}+\gamma \frac{d_{p+2}(d-1)^{3}(d-2)^{2}}{48 l^{8} r_{+}^{9-d}}\left(r_{+}^{2}+k l^{2}\right)^{3}\left((11 d-10) r_{+}^{2}-(5 d-22) k l^{2}\right)\right.} \\
& \left.-\left(2 \frac{d-2}{d-1}\left(\frac{d-3}{d-1}\right)^{\frac{d-3}{2}} l^{d-3}+\gamma \frac{d_{p+2}(d-2)^{4}}{3(d-1)}\left(\frac{d-3}{d-1}\right)^{\frac{d-9}{2}} l^{d-9}\right) \delta_{k,-1}\right]
\end{aligned}
$$

The free energy is then given by $F=I / \beta$. Inserting Eq. (55) into the action (59) and dividing by the inverse temperature $\beta$, we finally obtain the free energy $F(T)$ of the black hole in function of the temperature,

$$
\begin{aligned}
F(T)=-\frac{V_{d-2}}{16 \pi G_{d}} & {\left[\frac{r_{+(0)}^{d-1}(T)}{l^{2}}-k r_{+(0)}^{d-3}(T)+\frac{\gamma}{l^{6}} \frac{d_{p+2}(d-2)^{4}(d-1)^{3}}{48 l^{2} r_{+(0)}^{-d}(T)}\left(r_{+(0)}^{2}(T)+k l^{2}\right)^{4}\right.} \\
& \left.-\left(2 \frac{d-2}{d-1}\left(\frac{d-3}{d-1}\right)^{\frac{d-3}{2}} l^{d-3}+\gamma \frac{d_{p+2}(d-2)^{4}}{3(d-1)}\left(\frac{d-3}{d-1}\right)^{\frac{d-9}{2}} l^{d-9}\right) \delta_{k,-1}\right] .
\end{aligned}
$$

The corrected action (59), written in function of $r_{+}$, does not coincide with the expression $(35)+(10)$, obtained in a naive way. However, the free energy as a function of the temperature computed from the perturbed metric coincides with that obtained from (35), as was first observed in the cases $d=5, k=0$ in [19] and $d=5, k=1$ in [22]. As a consequence, also the correction to the entropy obtained from the perturbed metric coincides with the correction (36) computed from the unperturbed metric. This fact is particularly surprising in the $k=-1$ case, where the background used in the regularization receives itself modifications, and the subtraction has been done with respect to a different background. 
We now want to use the obtained thermodynamical quantities to determine the phase diagram, which is also relevant for the corresponding superconformal field theory via the AdS/CFT dictionary. To begin with, we study the case $d=5, k=1$, which corresponds to SYM on $S^{1} \times S^{3}$. In order to translate our results from the bulk to the SYM side, we will make use of the relation

$$
\frac{\alpha^{\prime}}{l^{2}}=\left(2 g_{Y M}^{2} N\right)^{-\frac{1}{2}}
$$

A first important thermodynamical information is obtained observing the behaviour of the inverse temperature $\beta$ in function of the black hole size $r_{+}$. The function $\beta\left(r_{+}\right)$has been plotted for different values of the string perturbation $\gamma$ in figure 1. We immediately see that
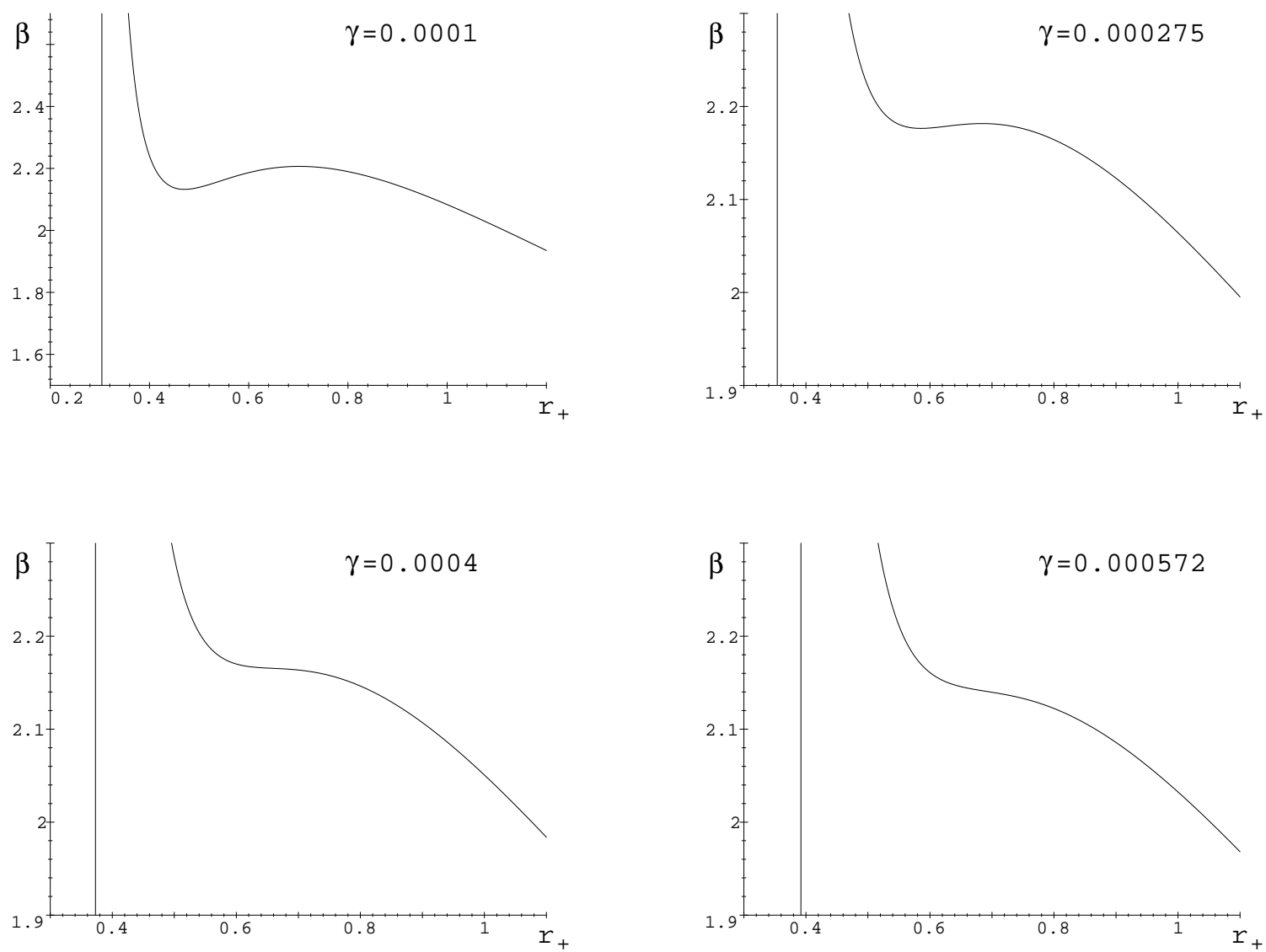

FIG. 1. Inverse temperature $\beta$ in function of the horizon position $r_{+}$for spherical black holes. A new branch of small stable black holes appears. For small $\gamma$ there are three branches; then, at $\gamma_{\text {infl }} \approx 0.0004$ a point of inflection appears, and for larger $\gamma$ the small and large black hole branches merge. (Here and in the following figures $\gamma$ is given in units of $l^{6}$ and $r_{+}$and $\beta$ are given in units of $l$.)

there exists a stable black hole corresponding to each value of the temperature, whereas in the unperturbed case there was a minimum temperature. This is due to the emergence of a stable branch of small black holes in the perturbed solution. Furthermore, there is 
a minimum size $r_{+}^{\text {min }}$ for stable black holes, where the temperature vanishes. For small $\gamma$, the small black holes are separated from the large black holes by a branch of unstable intermediate black holes. At $\gamma_{\text {infl }} \approx 0.0004$ the unstable branch degenerates to a point of inflection of $\beta\left(r_{+}\right)$, and for larger $\gamma$ the two stable branches merge together and no more distinction can be made beetween the small and large black hole phases. All this reminds us of the behaviour of a Van der Waals gas in the Clapeyron $(V, P)$ plane; and one could expect the point of inflection in $\beta(r)$ to signal a critical point. To go into this, however, we have to study the behaviour of the free energy, and take into consideration that in our system a third phase - AdS space filled with thermal radiation - is available. For charged AdS black holes, an analogous behaviour has recently been observed in 41,42].

In figure 2 we have reported the free energy of the perturbed solution as a function of $r_{+}$for different values of $\gamma$ (in all the figures the free energy $F$ is given in units of $V_{3} /\left(16 \pi G_{5}\right)$ ). To
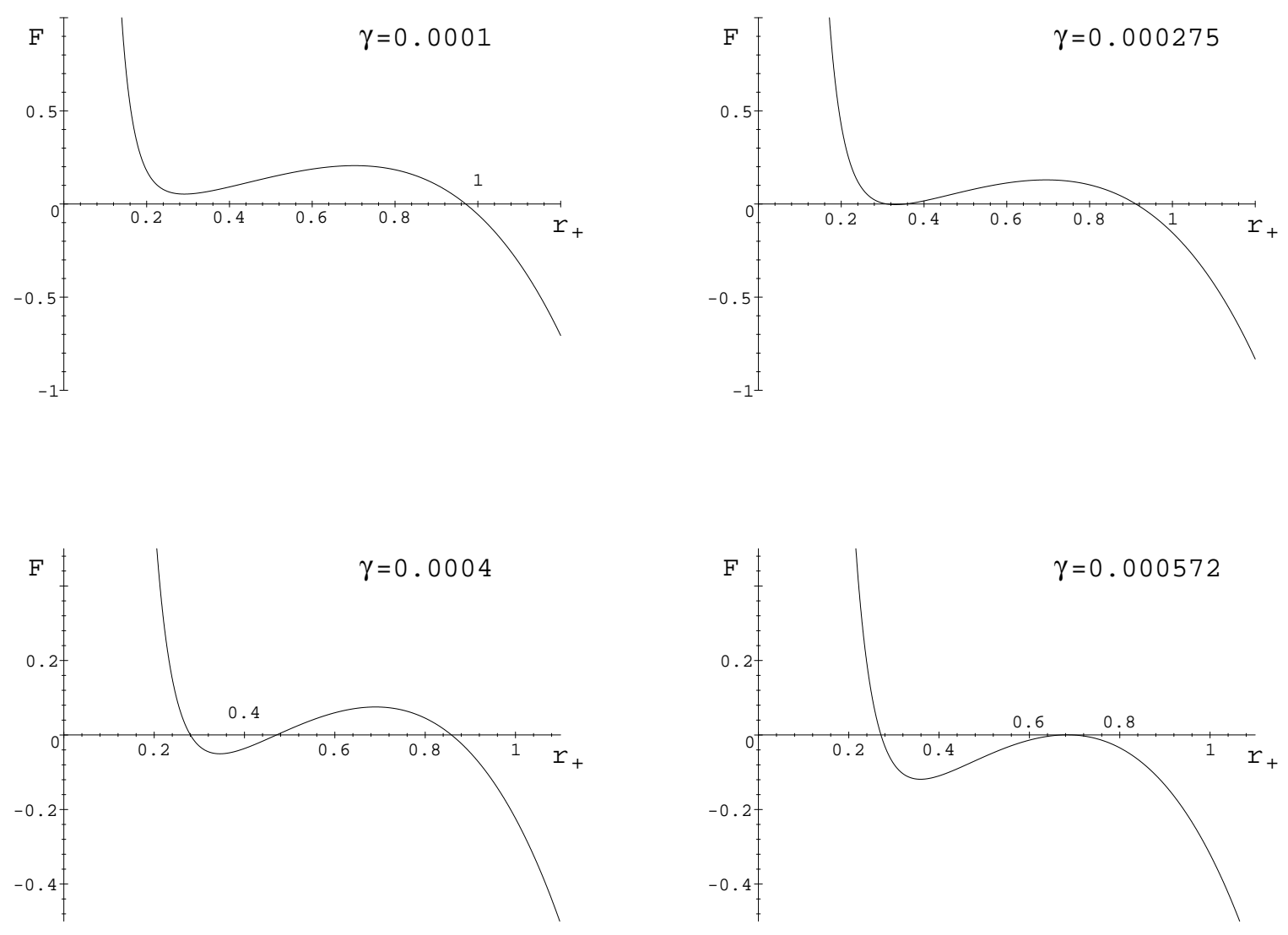

FIG. 2. Free energy $F$ in function of the horizon position $r_{+}$for spherical black holes. Two new critical values of $\gamma$ appear here: $\gamma_{1}^{\text {crit }} \approx 0.000275$, where the branch of small black holes begins to dominate over AdS space, and $\gamma_{2}^{\text {crit }} \approx 0.000572$, where the AdS phase disappears.

analyse these graphics, we must keep in mind that we computed the free energy with respect to AdS space, hence positive free energy means that $A d S$ space filled with thermal radiation is thermodynamically favoured, while negative free energy signals the collapse of the thermal 
radiation into a black hole (for a detailed analysis of stability and of the definition of the canonical ensemble, we refer to [42]). For small $\gamma$ we have the usual behaviour, with AdS space thermodynamically favoured with respect to the small black hole, while for sufficiently large $r_{+}$the canonical ensemble is dominated by the large black hole. At the critical value $\gamma_{1}^{\text {crit }} \approx 0.000275$ (corresponding to $g_{Y M}^{2} N \approx 33.4$ ) the free energy vanishes for $r_{+} \approx 0.36 l$, corresponding to the small black hole branch. For larger $\gamma$, we should distinguish four cases, but considering that there is a minimal size of the stable black holes, only three cases are relevant. We have first for small $r_{+}$a region where the small black hole dominates, then, as the size of the black hole grows, there is a transition to AdS space, and finally a second transition (the original Hawking-Page transition), to a large black hole phase. For $\gamma_{2}^{\text {crit }} \approx 0.000572$ (corresponding to $g_{Y M}^{2} N \approx 20.5$ ), the region of AdS-domination disappears and for larger $\gamma$ the black hole always dominates, if its size is greater that $r_{+}^{\min }$. Observe that, from the study of $\beta\left(r_{+}\right)$, the distinction between small and large black holes disappeared at $\gamma_{\text {infl }}<\gamma_{2}^{\text {crit }}$, whereas this distinction is reintroduced by the existence of a third phase, AdS space, and disappears definitively only at $\gamma_{2}^{\text {crit }}$.

We are ready now to examine the free energy as a function of $T$ and $\gamma$, from which we shall deduce the phase structure of our system. The graphs of $F(T)$ for different values of $\gamma$ are reported in figure 3. For $\gamma<\gamma_{1}^{\text {crit }}$ we have a phase dominated by AdS space filled with thermal radiation at low temperatures, and a phase dominated by large black holes at high temperatures, separated by the Hawking-Page transition. For $\gamma_{1}^{\text {crit }}<\gamma<\gamma_{2}^{\text {crit }}$, a new phase transition appears at low temperatures, separating a low-temperature phase of small black holes from an intermediate-temperature AdS phase. At high temperatures we have the Hawking-Page transition and again the large black hole dominates. Note that the critical temperature of this transition is lowered by $\gamma$-corrections, as first shown in [21. Finally, at $\gamma_{2}^{\text {crit }}$ the AdS phase disappears completely, and for higher $\gamma$ we have a unique phase dominated by black holes. For $\gamma>\gamma_{2}^{\text {crit }}$ the distinction between small and large black holes disappears; the system behaves like a liquid-vapour system. In the critical point $\left(\gamma_{2}^{\text {crit }}, T_{c} \approx 0.467 / l\right)$ we expect a higher order phase transition where the Hawking-Page phase transition disappears; this point has been identified by Gao and Li with the HorowitzPolchinsky correspondence point in 21]. The phase structure is summarized in the phase diagram of figure $⿴$. We stress the fact that the critical values $\gamma_{1}^{\text {crit }}$ and $\gamma_{2}^{\text {crit }}$ are very small (of the order of $10^{-4}$ ); as a consequence there should be no further significant corrections of higher order in $\gamma$ in the region of the phase diagram we explored. On the $\mathcal{N}=4 \mathrm{SYM}$ side, one has the correspondence point at $g_{Y M}^{2} N \approx 20.5$, which is considerably larger than the value of 1.65 obtained by Gao and Li. The reason is that they extrapolated the (steep) Hawking-Page transition curve to zero temperature, whereas we showed that this curve is interrupted by the intersection with the coexistence curve of small black holes and AdS space at a much smaller value of $\gamma$. Hence, in the SYM theory, the higher order phase transition is located at a value of the 't Hooft coupling where we have a better control on the large $N$ expansion.

We shall now turn to the $d=4$ and $d=7$ cases. The analysis of their thermodynamical behaviour can be carried over as in the $d=5$ case; as no further technical problems arise, we shall not report it here and instead discuss simply their phase structure.

The phase diagram for four-dimensional black holes is shown in figure 5. For non-zero $\gamma$ a new phase of small black holes appears at low temperature and dominates over AdS space 

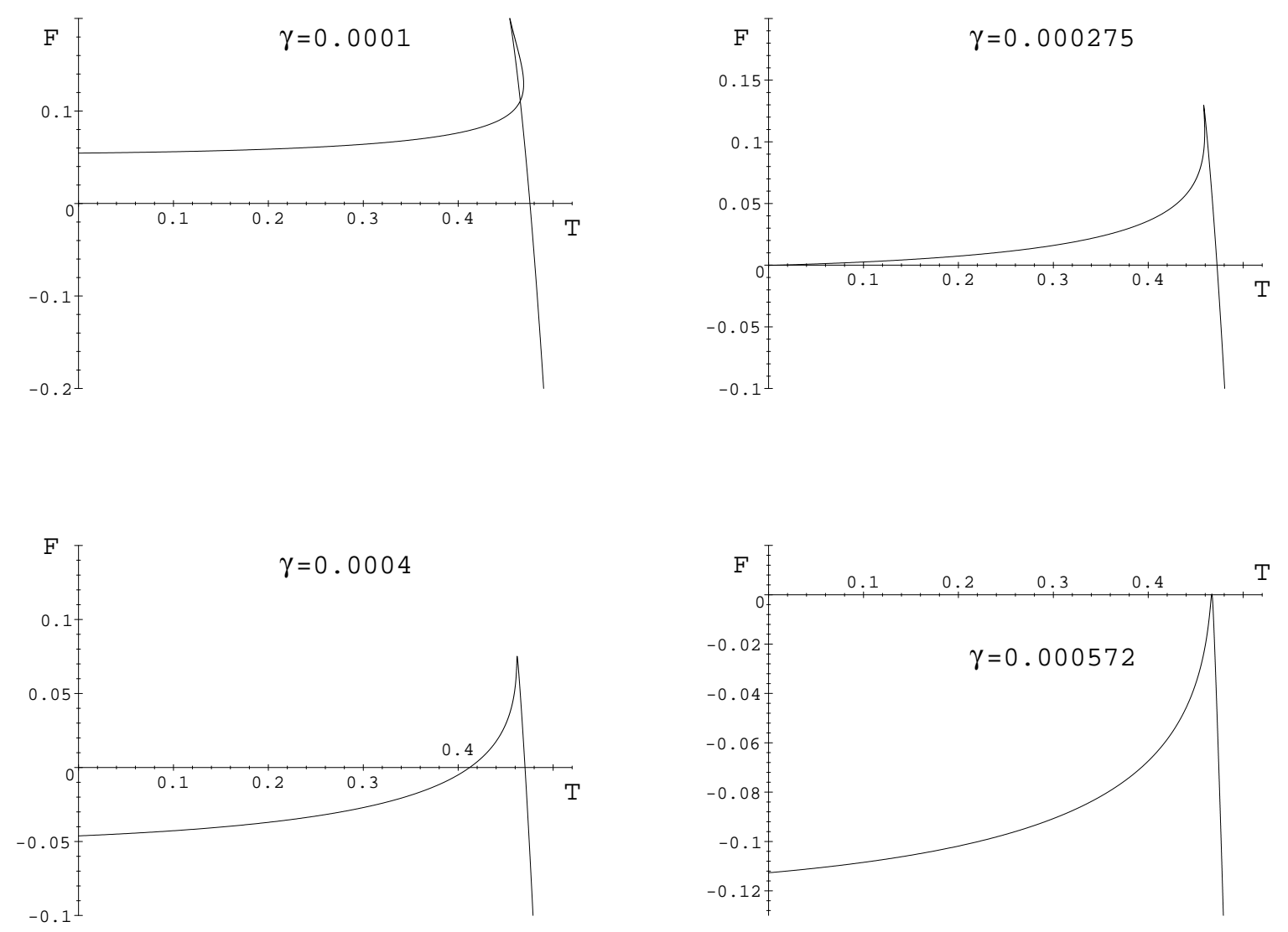

FIG. 3. Free energy $F$ in function of the temperature $T$ for spherical black holes. For $\gamma<\gamma_{1}^{\text {crit }} \approx 0.000275$ there is the standard Hawking-Page phase transition; at $\gamma_{1}^{\text {crit }}$ a new low-temperature phase of small black holes appears, and merges to the large black hole phase at $\gamma_{2}^{\text {crit }} \approx 0.000572$.

filled with thermal radiation. The low temperature phase is separated by a first order phase transition from an AdS phase. The coexistence curve small black hole/AdS terminates in the origin $\gamma=0, T=0$. At higher temperature we have the Hawking-Page phase transition between AdS and a phase dominated by a large black hole. Observe that the Hawking-Page temperature remains approximately constant as $\gamma$ grows. At $\gamma_{I I I}^{\text {crit }} \approx 3.07 \cdot 10^{-5}$ the two coexistence curves intersect for $T \approx 0.32 / l$ in a triple point. For higher $\gamma$ we are left with a small/large black hole phase separation curve, which continues more or less at the original Hawking-Page transition temperature; this curve could end in a second critical point like in the phase diagrams studied in [41], but in our perturbative approach we are not able to clarify this issue. From the AdS/CFT point of view, figure 5 corresponds to the phase structure of $\mathcal{N}=8$ super-Yang Mills theory on $S^{1} \times S^{2}$ with global $S O(8)$ R-symmetry, in the limit of sufficiently large $N$.

The seven-dimensional black hole case is somewhat different; AdS space always dominates over the small black hole. For this system, the phase diagram is drawn in figure 6. For 


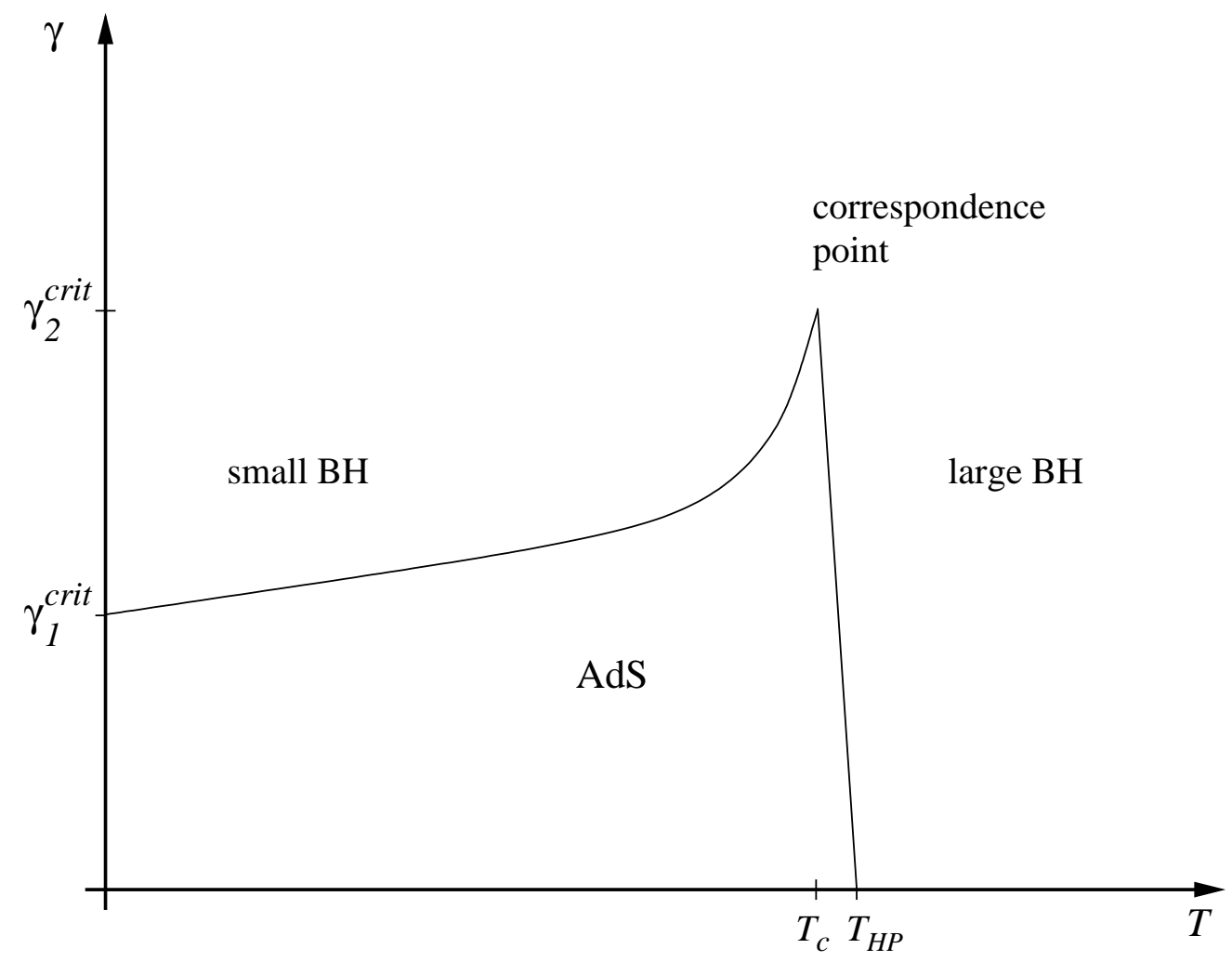

FIG. 4. Phase diagram for $\mathcal{N}=4$ SYM on $S^{1} \times S^{3}$ for sufficiently large 't Hooft coupling $g_{Y M}^{2} N$. For $\gamma<\gamma_{1}^{\text {crit }}$ AdS dominates over the small black holes and we have the usual Hawking-Page transition. Note that its critical temperature $T_{H P}$ decreases as $\gamma$ increases. At $\gamma_{1}^{\text {crit }} \approx 0.000275$, corresponding to $g_{Y M}^{2} N \approx 33.4$, the small black holes begin to dominate over AdS for small $T$, and we have three phases, a small black hole phase for small $T$, separated by an AdS phase from the large black hole phase. At $\gamma_{2}^{\text {crit }} \approx 0.000572$, corresponding to $g_{Y M}^{2} N \approx 20.5$, the two first order transitions degenerate to a higher order transition at $T_{c} \approx 0.467 / l$, and for larger $\gamma$ we are left with a unique black hole phase.

small $\gamma$ we have a low temperature AdS phase separated by a Hawking-Page transition from a high temperature black hole phase. The Hawking-Page transition temperature is lowered as $\gamma$ grows, and vanishes for $\gamma^{\text {crit }} \approx 5.48 \cdot 10^{-5}$; for higher $\gamma$ only the black hole phase remains. From the boundary conformal field theory point of view, the spherical seven-dimensional AdS black hole in M-theory compactified on a four-sphere corresponds to an exotic six-dimensional theory with $(0,2)$ supersymmetry. The flat analogue of this theory is particularly interesting; Witten [3] suggested that with a supersymmetry-breaking compactification to four dimensions it may be possible to explore four-dimensional $S U(N)$ Yang-Mills theory in the large $N$ limit; this theory was therefore considered one of the main approaches to confinement in QCD.

Finally, for the hyperbolic or flat cases, $k=-1$ or $k=0$, one checks that the corrected free energy $F(T)$ is always negative for positive $T$; it is zero only for the background, i. e. for the extremal black hole with $T=0$. This means that the (deconfining) black hole phase dominates for all temperatures, also if one takes the leading stringy (or elevendimensional Planck length) corrections into account. So these do not cause any phase 


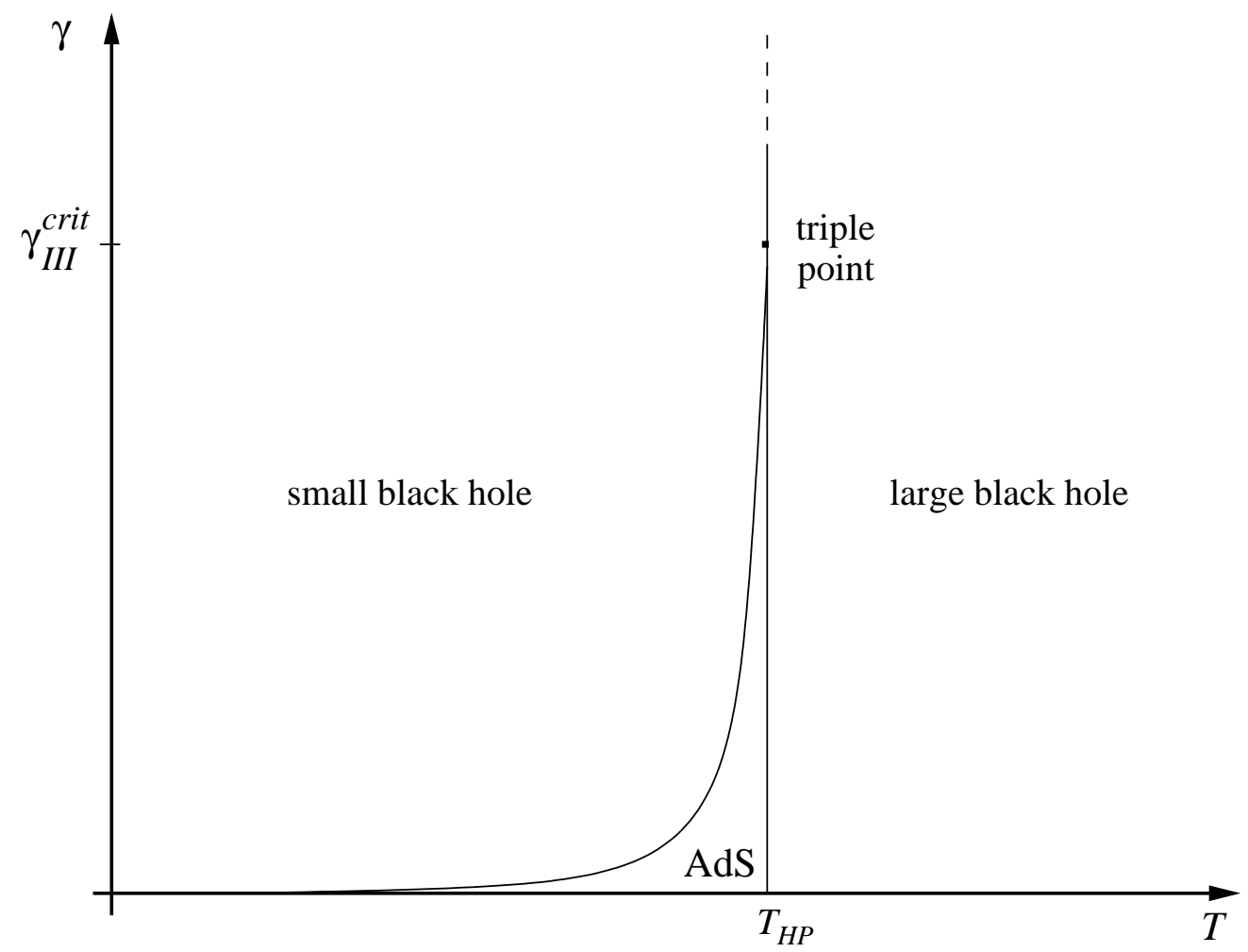

FIG. 5. Phase diagram for $\mathcal{N}=8 \mathrm{SYM}$ on $S^{1} \times S^{2}$ with global $S O(8)$ R-symmetry. For $0<\gamma<\gamma_{I I I}^{\text {crit }}$ there is a small black hole phase for small $T$, separated by an AdS phase from the large black hole phase. At $\gamma_{I I I}^{\text {crit }} \approx 3.07 \cdot 10^{-5}$, the AdS phase disappears, leaving a triple point $\left(T_{c} \approx 0.32 / l\right)$. For higher $\gamma$ we have a first order transition between a small and a large black hole phase.

transition. However, if the transverse manifold (i. e. $\mathbb{H}^{p}$ for $k=-1$ and $\mathbb{R}^{p}$ for $k=0$ ) is compactified, it supports non-contractible 1-cycles around which strings can wind. When the length of these cycles become comparable to the string scale $\ell_{s}$, the winding modes become very light, and we expect the geometry to be modified. Now for the hyperbolic black hole there is a minimum horizon radius $r_{0}$ given by (6). The length of the noncontractible cycles in general depends on several parameters, like the moduli and genus in the case of a compact Riemann surface $(p=2)$. If we neglect for the moment this dependence and suppose those lengths to be of order one, light winding modes become important for $r_{0} \sim \ell_{s}$, which gives a rather large value of $\alpha^{\prime}$. The behaviour is different for the toroidal black hole however, as here the horizon radius can become arbitrarily small, it shrinks to zero size in the extremal case $T=0$. So light winding modes can modify the geometry already for small values of $\alpha^{\prime}$. These finite size effects have been discussed in [26]. Let us take a short look on what happens when the moduli of the torus are taken into account. As a model we take the (uncorrected) black hole metric in IIB supergravity,

$$
d s^{2}=-f(r) d t^{2}+f(r)^{-1} d r^{2}+r^{2}\left(d x^{2}+|\tau|^{2} d y^{2}+2 \operatorname{Re}(\tau) d x d y+d z^{2}\right)+l^{2} d \Omega_{5}^{2},
$$

where 


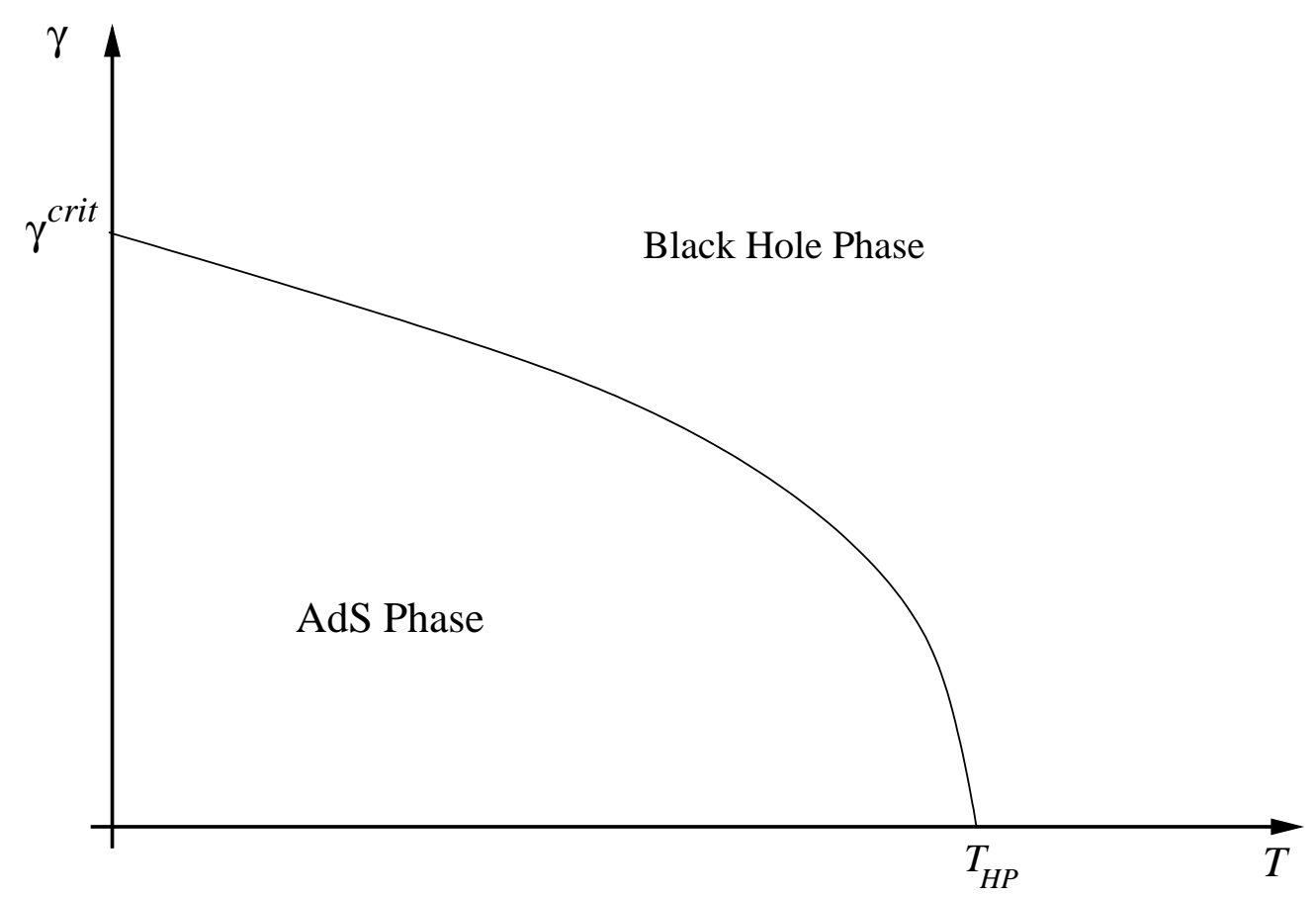

FIG. 6. Phase diagram for $(0,2)$ CFT on $S^{1} \times S^{5}$. For $\gamma<\gamma^{c r i t}$, AdS dominates over the black hole and we have the usual Hawking-Page transition. However $T_{H P}$ decreases as $\gamma$ increases. At $\gamma^{\text {crit }} \approx 5.48 \cdot 10^{-5}$, the Hawking-Page transition disappears, leaving a unique black hole phase for higher $\gamma$.

$$
f(r)=-\frac{\eta}{r^{2}}+\frac{r^{2}}{l^{2}}
$$

and $x, y, z \in[0,1]$. For the sake of simplicity, we restrict ourselves to one complex modulus $\tau$. The fundamental cycles in $x$ or $z$ direction have length 1 , while those in $y$ direction have length $|\tau|$. Winding modes along $x, z$ then become important for $r_{+} \sim \ell_{s}$, and winding modes along $y$ for $r_{+} \sim \ell_{s} /|\tau|$. Note that these two lengths can differ considerably, if $|\tau| \gg 1$ or $|\tau| \ll 1$. Let us assume e. g. the latter case. Then for $r \sim \ell_{s} /|\tau|$ one should go over to a T-dual language (T-duality along $y$ ). The crossover temperature is

$$
T=T_{1} \equiv \frac{\ell_{s}}{\pi l^{2}|\tau|}
$$

For $r \sim \ell_{s}$ one has to perform another T-duality along $x, z$, where now the corresponding crossover temperature is $T=T_{2} \equiv T_{1}|\tau| \ll T_{1}$. In each of the three regimes, $0 \leq T \leq T_{2}$, $T_{2} \leq T \leq T_{1}$, and $T>T_{1}$, one now has to examine the thermodynamic stability of the corresponding manifold, as has been done in [26] for $\tau=i$, i. e. for $T_{1}=T_{2}$. In this case, the authors of [26] argued that there is a phase transition at a temperature

$$
T_{\text {trans }}=\left(2 g_{Y M}^{2} N\right)^{-\frac{1}{4}} T_{1}=\frac{\ell_{s}}{l} T_{1},
$$

below which the dual $\tilde{X}_{b h}$ of the black hole manifold $X_{b h}(62)$, which represents a system of D0-branes smeared over the torus $T^{3}$ (cf. [26] for the T-dual metric), goes over to a system of 
D0-branes localized on the $T^{3}$. This phase transition is the toroidal analogue of the HawkingPage transition which occurs for spherical black holes. If the modulus $|\tau|$ is very different from $i$ (so that e. g. $T_{1} \gg T_{2}$ ), we expect a more complicated phase diagram, showing perhaps more than one phase transition. (A full thermodynamical discussion involving the determination of the phase diagram would go beyond the scope of this paper, so we leave this issue for future investigations). In particular, we expect a rather complicated phase diagram in the moduli space of the torus.

\section{SUMMARY AND DISCUSSION}

In the present paper, we computed stringy or M-theory corrections to $d$-dimensional anti-de Sitter black holes for $d=4,5,7$. Thereby we allowed for spherical, flat or hyperbolic event horizons. In order to obtain the corrections to the black hole thermodynamics, we first used a simplified perturbative approach, which consists in plugging the unperturbed metric into the term quartic in the Weyl tensor appearing in the modified supergravity action. Then also the corrected black hole metrics have been determined explicitely in the various cases. Quite surprisingly, the resulting thermodynamical quantities, like free energy or entropy, coincide with those obtained by the above mentioned simplified approach. This coincidence holds for all values of $d$ and for all event horizon topologies under consideration. Especially in the hyperbolic case, this agreement is remarkable, as the calculation of the action for hyperbolic black holes involves a nontrivial background subtraction. For black holes with flat or hyperbolic event horizons, we found that the first stringy or M-theory corrections do not give rise to any phase transition; the system is in the black hole phase for any temperature. If the spatial black hole geometry supports noncontractible 1-cycles, i. e. if one compactifies the $\mathbb{R}^{p}$ or the $\mathbb{H}^{p}$, we have winding modes which become very light if the length of these 1-cycles is of order the string scale. Then we expect the geometry to be modified. For hyperbolic black holes, which have a minimum event horizon radius of the order the AdS scale $l$, these winding modes in general become light only for a large value of $\alpha^{\prime}$. (Taking into account the moduli of the compact space could change this behaviour, but we did not consider them here). For toroidal black holes however, there is no minimum event horizon radius, so already for small values of $\alpha^{\prime}$ light winding modes can become important. As these finite size effects have already been discussed in [26] for symmetric tori, we limited ourselves to a short discussion of the nonsymmetric case, indicating the appearance of a rather complicated phase diagram.

As for spherical black holes, which are relevant to study SYM on $S^{1} \times S^{p}$, we showed that the stringy or M-theory corrections give rise to the emergence of a stable branch of small black holes. By considering the free energy, the corresponding SYM phase diagram has been determined for $d=5$ in the limit of sufficiently large 't Hooft coupling, where the restriction to the $\mathcal{O}\left(\alpha^{\prime 3}\right)$ corrections is valid. The phase diagram shows that the HawkingPage transition temperature decreases with increasing string scale, and finally disappears at a value of $g_{Y M}^{2} N \approx 20.5$, which is considerably larger than the estimate given by Gao and $\mathrm{Li}$ 21]. This critical point in the phase diagram has been identified by the authors of [21] with the correspondence point of Horowitz and Polchinski. We found that there is a second critical value of the 't Hooft coupling in the $S^{1} \times S^{3}$ SYM phase diagram, namely 
$g_{Y M}^{2} N \approx 30.4$. Above this value, we have the confining phase of thermal AdS space for low temperatures and the deconfining black hole phase for sufficiently high temperatures. For $20.5<g_{Y M}^{2} N<30.4$, one has a phase of small black holes for low temperatures, an AdS phase in an intermediate temperature regime, and finally a phase of large black holes for high temperatures. For $g_{Y M}^{2} N<20.5$, the system is always in the black hole phase; besides, the small and large black holes have become indistinguishable. The $d=4$ black hole, corresponding to $\mathcal{N}=8 \mathrm{SYM}$ on $S^{1} \times S^{2}$, has a somewhat different phase structure. The phase of small black holes now already appears for any nonzero value of $\gamma$. Furthermore, the critical point is a triple point. Above it, no AdS phase appears, but there is still a small/large black hole phase transition. Finally, for $d=7$ (corresponding to an exotic $(0,2)$ CFT on $S^{1} \times S^{5}$ ), there is no small black hole phase, and the AdS phase is located in the region of small temperatures and small values of $\gamma$.

\section{ACKNOWLEDGEMENTS}

The authors would like to thank G. Cognola, F. Ferrari, M. Porrati and L. Vanzo for helpful discussions.

The part of this work due to D. K. has been supported by a research grant within the scope of the Common Special Academic Program III of the Federal Republic of Germany and its Federal States, mediated by the DAAD. 


\section{REFERENCES}

[1] J. M. Maldacena, The Large N Limit of Superconformal Field Theories and Supergravity, Adv. Theor. Math. Phys. 2 (1998) 231.

[2] E. Witten, Anti-de Sitter Space And Holography, Adv. Theor. Math. Phys. 2 (1998) 253.

[3] E. Witten, Anti-de Sitter Space, Thermal Phase Transition, And Confinement In Gauge Theories, Adv. Theor. Math. Phys. 2 (1998) 505.

[4] S. S. Gubser, I. R. Klebanov, and A. M. Polyakov, Gauge Theory Correlators from Non-Critical String Theory, Phys. Lett. B428 (1998) 105.

[5] S. W. Hawking and D. N. Page, Thermodynamics of Black Holes in Anti-de Sitter Space, Commun. Math. Phys. 87 (1983) 577.

[6] A. Kehagias and K. Sfetsos, On running couplings in gauge theories from type-IIB supergravity, Phys. Lett. B454 (1999) 270.

[7] S. S. Gubser, Dilaton-driven Confinement, hep-th/9902155.

[8] L. Girardello, M. Petrini, M. Porrati, and A. Zaffaroni, Confinement and Condensates without fine tuning in Supergravity Duals of Gauge Theories, JHEP 9905 (1999) 026.

[9] I. R. Klebanov, From Threebranes to Large N Gauge Theories, hep-th/9901018.

[10] S. W. Hawking, Black Holes in General Relativity, Commun. Math. Phys. 25 (1972) 152.

[11] S. Åminneborg, I. Bengtsson, S. Holst, and P. Peldán, Making Anti-de Sitter Black Holes, Class. Quantum Grav. 13 (1996) 2707.

[12] R. B. Mann, Black Holes of Negative Mass, Class. and Quantum Grav. 14 (1997) 2927;

R. B. Mann, Topological Black Holes - Outside Looking In, gr-qc/9709039 (1997);

W. L. Smith and R. B. Mann, Formation of Topological Black Holes from Gravitational Collapse, Phys. Rev. D 56 (1997) 4942.

[13] J. P. S. Lemos, Two-Dimensional Black Holes and Planar General Relativity, Class. and Quantum Grav. 12 (1995) 1081;

J. P. S. Lemos, Cylindrical Black Hole in General Relativity, Phys. Lett. B353 (1996) 46 ;

J. P. S. Lemos and V. T. Zanchin, Rotating Charged Black Strings in General Relativity, Phys. Rev. D 54 (1996) 3840.

[14] R. G. Cai and Y. Z. Zhang, Black Plane Solutions in Four Dimensional Spacetimes, Phys. Rev. D 54 (1996) 4891.

[15] C. G. Huang and C. B. Liang, A Torus-Like Black Hole, Phys. Lett. A201 (1995) 27;

C. G. Huang, Acta Phys. Sin. 4 (1996) 617.

[16] L. Vanzo, Black Holes with Unusual Topology, Phys. Rev. D 56 (1997) 6475.

[17] D. Birmingham, Topological Black Holes in Anti-de Sitter Space, Class. Quant. Grav. 16 (1999) 1197.

[18] R. Emparan, AdS Membranes Wrapped on Surfaces of Arbitrary Genus, Phys. Lett. B432 (1998), 74.

[19] S. S. Gubser, I. R. Klebanov and A. A. Tseytlin, Coupling Constant Dependence in the Thermodynamics of $\mathcal{N}=4$ Supersymmetric Yang-Mills Theory, Nucl. Phys. B534 (1998) 202. 
[20] J. Pawelczyk and S. Theisen, $A d S_{5} \times S^{5}$ Black Hole Metric at $\mathcal{O}\left(\alpha^{\prime 3}\right)$, JHEP 09 (1998) 010.

[21] Y. Gao and M. Li, Large N Strong/Weak Coupling Phase Transition and the Correspondence Principle, Nucl. Phys. B551 (1999) 229.

[22] K. Landsteiner, String Corrections to the Hawking-Page Phase Transition, Mod. Phys. Lett. A14 (1999) 379.

[23] G. T. Horowitz and J. Polchinski, A Correspondence Principle for Black Holes and Strings, Phys. Rev. D 55 (1997) 6189.

[24] M. Li, E. Martinec, and V. Sahakian, Black Holes and the SYM Phase Diagram, Phys. Rev. D 59 (1999) 044035.

[25] E. Martinec and V. Sahakian, Black Holes and the SYM Phase Diagram. II, Phys. Rev. D 59 (1999) 124005.

[26] J. L. F. Barbón, I. I. Kogan, and E. Rabinovici, On Stringy Thresholds in SYM/AdS Thermodynamics, Nucl. Phys. B544 (1999) 104.

[27] A. A. Tseytlin, Black Holes and Exact Solutions in String Theory, Proc. of the International School of Astrophysics "D. Chalonge", 4-16 Sept. 1994, Erice, ed. N. Sanchez (World Scientific, Singapore).

[28] E. Witten, String theory dynamics in various dimensions, Nucl. Phys. B443 (1995) 85.

[29] M. J. Duff, H. Lü and C. N. Pope, $A d S_{5} \times S^{5}$ Untwisted, Nucl. Phys. B532 (1998), 181.

[30] M. J. Duff, H. Lü and C. N. Pope, Supersymmetry without supersymmetry, Phys. Lett. B409 (1997) 136.

[31] D. Birmingham, String Theory Formulation of anti-de Sitter Black Holes, Phys. Lett. B428 (1998) 263.

[32] E. Bergshoeff, C. Hull and T. Ortín, Duality in the type-II superstring effective action, Nucl. Phys. B451 (1995) 547.

[33] M. J. Duff, B. E. W. Nilsson and C. N. Pope, Kaluza-Klein supergravity, Phys. Rep. 130 (1986) 1.

[34] E. Halyo, Supergravity on $A d S_{4 / 7} \times S^{7 / 4}$, JHEP 04 (1998) 011,

E. Halyo, Supergravity on AdS $S_{5 / 4} \times$ Hopf Fibrations and Conformal Field Theories, hepth/9803193.

[35] M. T. Grisaru and D. Zanon, Sigma-Model Superstring Corrections to the EinsteinHilbert Action, Phys. Lett. B177 (1986) 347;

M. D. Freeman, C. N. Pope, M. F. Sohnius, and K. S. Stelle, Higher-Order Sigma Model Counterterms and the Effective Action for Superstrings, Phys. Lett. B178 (1986) 199; Q. -H. Park and D. Zanon, More on Sigma Model beta-Functions and Low-Energy Effective Actions, Phys. Rev. D 35 (1987) 4038.

[36] D. J. Gross and E. Witten, Superstring Modifications of Einstein Equations, Nucl. Phys. B277 (1986) 1.

[37] A. A. Tseytlin, Ambiguity in the Effective Action in String Theories, Phys. Lett. B176 (1986) 92.

[38] G. T. Horowitz and D. L. Welch, Exact Three Dimensional Black Holes in String Theory, Phys. Rev. Lett. 71 (1993) 328.

[39] R. Kallosh and A. Rajaraman, Vacua of M-Theory and String Theory, Phys. Rev. D 58 (1998) 125003.

[40] M. M. Caldarelli and D. Klemm, Supersymmetry of anti-de Sitter black holes, 
Nucl. Phys. B545 (1999) 434.

[41] A. Chamblin, R. Emparan, C. V. Johnson and R. C. Myers, Charged AdS Black Holes and Catastrophic Holography, hep-th/9902170.

[42] M. Cvetič and S. S. Gubser, Phases of R-charged Black Holes, Spinning Branes and Strongly Coupled Gauge Theories, JHEP 9904 (1999) 024. 